\title{
Effect of microstructure on the formability of Ti21S alloy
}

Cai Hu, Lionel Leotoing, Philippe Castany, Dominique Guines and Thierry Gloriant

Cai Hu. Univ Rennes, INSA Rennes, LGCGM-EA 3913, Rennes, France

Lionel Leotoing. Univ Rennes, INSA Rennes, LGCGM-EA 3913, Rennes, France

Corresponding author: LEOTOING Lionel. E-mail address: Lionel.Leotoing@insa-rennes.fr

Philippe Castany. Univ Rennes, INSA Rennes, CNRS, ISCR UMR 6226, Rennes, France

Dominique Guines. Univ Rennes, INSA Rennes, LGCGM-EA 3913, Rennes, France

Thierry Gloriant. Univ Rennes, INSA Rennes, CNRS, ISCR UMR 6226, Rennes, France

Abstract. Titanium alloys find a wide range of uses, especially in the aeronautic industry because of a combination of favorable specifications in terms of strength-to-weight ratio, corrosion resistance and performance at high temperature. If many works are interested in mechanical properties, as well as microstructure, few of them studied the effect of microstructure on formability. The aim of this work is to study the influence of the microstructure on the formability of $\beta$ metastable titanium alloys (Ti21S) which are increasingly used in aeronautical applications. For this purpose, two different heat treatments are performed on Ti21S alloy in order to propose different microstructures. Based on uniaxial tensile tests, the elastoplastic hardening behavior and the limit strain in the uniaxial tension state are obtained and allow to determine one point of the forming limit curve (FLC). From these experimental observations, it is shown that the microstructure has an important effect on the formability: precipitation of $\alpha$ phase reduces the formability in comparison with full $\beta$ phase microstructure. Finally, a finite element M-K model is used and calibrated to predict the whole FLC for the different investigated microstructures.

Keywords. Titanium Alloys, Microstructure, Formability, Predictive Forming Limit Curve

\section{Introduction}

Ti21S is a metastable $\beta$ titanium alloy that has wide use in the aviation industry. It exhibits high strength, good resistance to corrosion, and good performance at high temperature. Sheet forming process is commonly used in the manufacturing of many components in aerospace [1]. The onset of necking during forming is usually described by the Forming Limit Curve (FLC). This curve is a standard method to characterize the formability of sheet metals and is used to avoid the failure of materials while the manufacturing process [2][3]. The experimental determination of forming limit curves is complex and two tests are generally performed: out-of-plane stretching such as Nakazima test [4] and in-plane stretching such as Marciniak test [5]. M-K model is also presented as an analytical method to calculate the forming limit curves [6].

The processing of materials can affect the mechanical properties, and then modify the forming limit curve through the change of microstructure. The mechanical anisotropy is also proved to have a main influence on formability [7]. As an example, cross rolling reduces the anisotropy and then improves the formability of pure titanium [8]. $\beta$ titanium alloys show good formability in the solution heat treated and quenched state, but it is reduced after aging because of $\alpha$ phase precipitation that improves the strength and reduces the ductility [9]. Data about FLC of $\beta$ titanium alloys are scarcely reported [10] while this knowledge is crucial to predict the forming of such alloys. The link between FLC and microstructure was also never investigated in $\beta$ titanium alloys.

This work aims to study the formability of the Ti21S alloy with two different microstructures obtained after different heat treatments. Tensile tests on two different microstructures and several directions from the rolling one are then conducted to obtain mechanical properties, including the Lankford coefficients. A numerical M-K model is used to predict forming limit points from the results of tensile tests. Then the complete predicting FLC can be plotted after 
Effect of microstructure on the formability of Ti21S alloy

calibrating the M-K model to investigate the effect of microstructure.

\section{Experimental procedure}

The as-received Ti21S rolled plate has a composition of Ti-15Mo-2.7Nb-3Al-0.2Si (wt. \%) and a thickness of $0.8 \mathrm{~mm}$. A solution treatment at $900^{\circ} \mathrm{C}$ (above $\beta$ transus temperature) for $30 \mathrm{~min}$, followed by water quenching is first performed. An aging treatment in the $\alpha+\beta$ domain at $650^{\circ} \mathrm{C}$ for 8 hours followed by furnace cooling is made for some of the solution treated pieces. Tensile specimens with a central gauge of $45 \times 5 \mathrm{~mm}^{2}$ are machined for these 2 conditions (Fig. 1 a): solution treated and quenched state (Ti21S ST) and solution treated and aged state (Ti21S STA). In order to investigate the effect of anisotropy, tensile specimens are cut along the rolling direction (L) and the transverse direction (T). Specimens are labeled with the tensile orientation followed by their heat treatment condition (Ti21S L,ST and Ti21S T,ST as examples). Supplemental specimens cut with an angle of $45^{\circ}$ to the rolling direction of the Ti21S ST condition are also tested in order to calculate anisotropy yield stress ratios. For each condition, 3 to 5 specimens are tested in order to ensure the representativeness of results. Tensile tests are carried out on INSTRON-3345 testing machine at room temperature with an initial strain rate of $10^{-3} \mathrm{~s}^{-1}$. A random pattern is spayed on the surface of the sample and a camera is set for filming this specimen surface during the tensile test in order to calculate in-plane deformations (Fig. 1).

a) Tensile specimen and its dimensions ( $\mathrm{mm})$

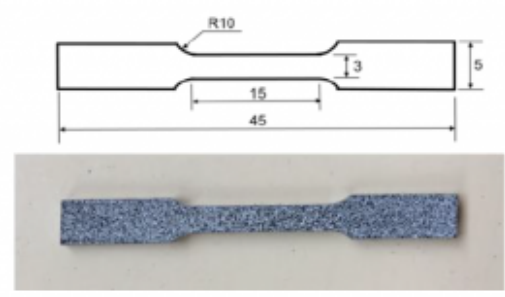

b) Tensile device

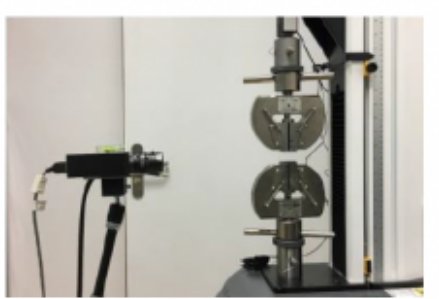

Fig. 1 Tensile specimens and device.

Uniaxial tensile tests are also used to determine one forming limit point on the forming limit diagram. In order to precisely measure the deformation, Digital Image Correlation (DIC) is conducted on the tensile specimens with the GOM software. The evolution of deformation in three sections (Fig. 2 a), along the longitudinal direction of the specimens, can be plotted just before rupture. Due to the necking, a rising part on the curve is observed. Just after, rupture happens at the position with the highest major deformation. Two inner boundary points A and B (Fig. 2 b) are defined according to the position of the necking zone. Besides, the width $\left(\mathrm{W}_{\mathrm{l}}\right.$ and $\mathrm{W}_{\mathrm{r}}$ ) of two fit windows needs to be adjusted. Then, an inverse parabola of a quadratic equation is used to fit the points in the two fit windows. The highest point on the inverse parabola gives the limit major strain $\varepsilon_{1}$. The corresponding limit minor strain $\varepsilon_{2}$ is calculated by following the strain path in the necking zone. For each specimen, the average forming limit strains are calculated with the three sections. By this method, a limit point can be added to the forming limit diagram. 

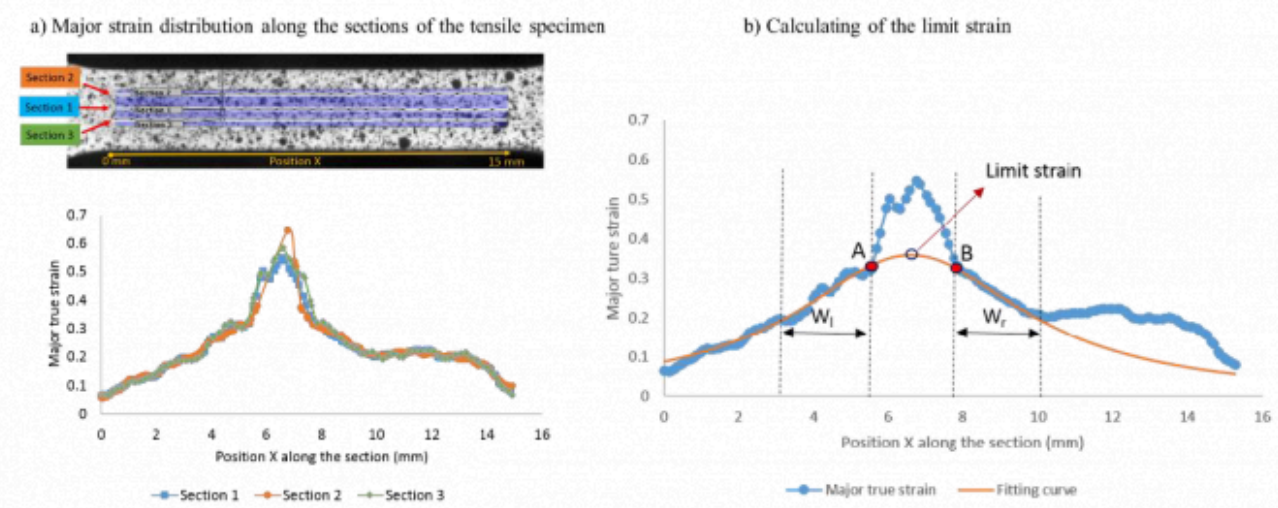

Fig. 2 Method of detecting forming limit point.

Lankford coefficients $\left(r_{\theta}\right)$ are also evaluated by the DIC method. 5 points along the previous sections are chosen. Strain components $\varepsilon_{1}$ and $\varepsilon_{2}$ along longitudinal and transverse directions are calculated. These Lankford coefficients are calculated over a range of about $50 \%$ to $75 \%$ of $\varepsilon_{\max }$ (Fig. 3 a). Incompressibility equation $\left(\varepsilon_{1}+\varepsilon_{2}+\varepsilon_{3}=0\right)$ is used to calculate the strain component $\varepsilon_{3}$ through the specimen thickness. Then, the curve $\varepsilon_{2}$ versus $\varepsilon_{3}$ can be plotted for each point as shown in Fig. $3 \mathrm{~b}$ where an average over the 5 chosen points has been performed. The slope of the fitting line of the average curve $\varepsilon_{2}$ versus $\varepsilon_{3}$ is the $r$ value (Fig. 3 b). This method significantly reduces the noise when the deformation is small. Values of $r_{0}, r_{45}, r_{90}$ are calculated through the tensile specimens with the angle of $0^{\circ}, 45^{\circ}$, and $90^{\circ}$ to the rolling direction.

a) The 5 points chosen and the $\varepsilon_{\max }$ range considered

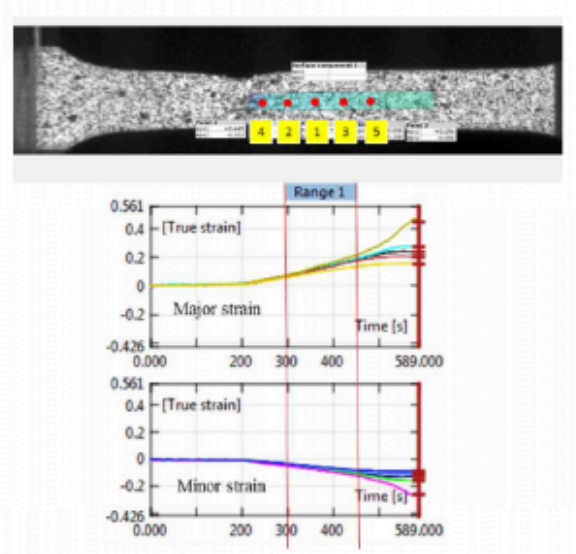

b) $\varepsilon_{2}$ versus $\varepsilon_{3}$ for Ti2 1 S L,ST.

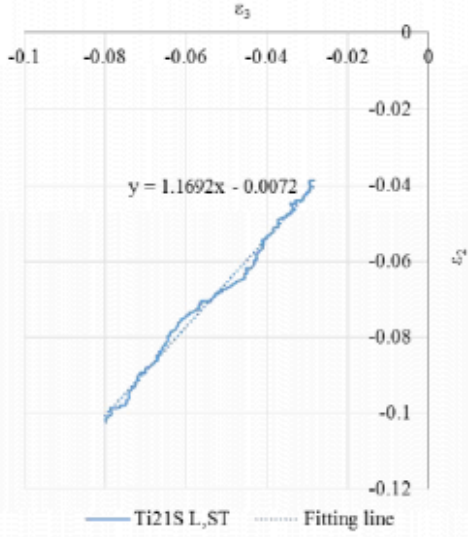

Fig. 3 Method of calculating $r$ value.

\section{Results and discussions}

\subsection{Mechanical properties}

The comparison of tensile curves of Ti21S ST and Ti21S STA along L and T directions is shown in Fig. 4. The Young's 
Effect of microstructure on the formability of Ti21S alloy

modulus (E), Yield Strength (Rpo.2), Ultimate Tensile Strength (UTS), Elongation (A), and their Standard deviations ( $\bar{\sigma}$ ) are summarized in Table. 1.

For Ti21S ST specimens, each value along $\mathrm{L}$ direction is higher than along T direction, except for the Young's modulus, which is nearly the same for both directions. On the contrary, Ti21S STA specimens do not show this phenomenon. This observation highlights an anisotropy in mechanical properties for the ST condition.

After the aging process, Ti21S STA specimens show an increased tensile strength compared to the Ti21S ST condition. The ultimate tensile strength is increased from $989 \mathrm{MPa}$ to $1304 \mathrm{MPa}$ (L), or from $890 \mathrm{MPa}$ to $1300 \mathrm{MPa}$ (T). Young's modulus also increased evidently. But the elongation of Ti21S STA is less than 10\%, while Ti21S ST has 16\% and $27 \%$. The images of fractured specimens in Fig. 4 show that there is merely no necking near the crack for STA specimens, while few necking appears in ST specimens. The cracks are also perpendicular to the rolling direction for STA specimens. All these features of STA specimens are due to the well-known hardening effect and fragilizing effect of $\alpha$ phase precipitation [11].

a) Tensile curve of Ti21S L,ST and Ti21S L,STA

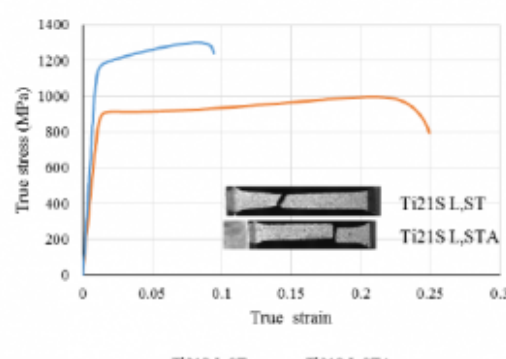

- Ti215 LST - T21SLSTA b) Tensile curve of Ti21S T,ST and Ti21S T,STA

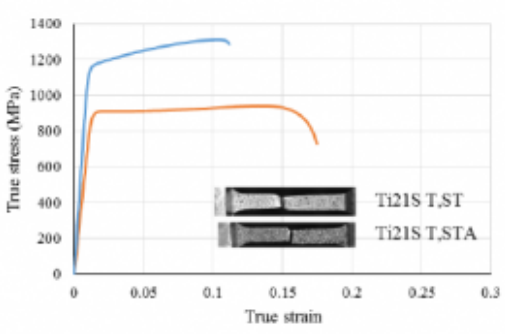

- TI21S T.ST - T21S T.STA

Fig. 4 Comparison of tensile curves; insets show typical specimens after fracture for each condition.

Table. 1 Young's modulus (E), Yield Strength (Rp 0.2$)$, Ultimate Tensile Strength (UTS), Elongation (A) and their Standard deviation $(\bar{\sigma})$ of Ti21S ST and Ti21S STA.

\begin{tabular}{ccccccccc}
\hline Specimen & $\mathrm{E}(\mathrm{GPa})$ & $\bar{\sigma}(\mathrm{E})(\mathrm{GPa})$ & $\mathrm{Rp}_{02}(\mathrm{MPa})$ & $\bar{\sigma}\left(\mathrm{Rp} 0_{2}\right)(\mathrm{MPa})$ & $\mathrm{UTS}(\mathrm{MPa})$ & $\bar{\sigma}(\mathrm{UTS})(\mathrm{MPa})$ & $\mathrm{A}(\%)$ & $\bar{\sigma}(\mathrm{A})(\%)$ \\
\hline Ti21S L,ST & 75 & 3 & 894 & 24 & 989 & 19 & 28 & 1.7 \\
\hline Ti21S T,ST & 71 & 3 & 867 & 28 & 890 & 29 & 16 \\
\hline Ti21S L,STA & 122 & 4 & 1167 & 3 & 1304 & 7 & 10 \\
\hline Ti21S T,STA & 113 & 4 & 1176 & 26 & 1300 & 23 & 9.8 \\
\hline
\end{tabular}

In order to characterize more precisely the mechanical anisotropy of the Ti21S ST material, $r$-values are calculated and are shown in Table. 2. The $r$-value at $45^{\circ}$ is lower than the others and $r 90$ is slightly higher than $r_{0}$.

Table. 2 The $r$-values of Ti21S ST. 


\begin{tabular}{cccc}
\hline Specimen & $r_{0}$ & $r_{45}$ & $r_{90}$ \\
\hline Ti21S ST & 1.04 & 0.69 & 1.17 \\
\hline
\end{tabular}

\subsection{Formability}

The result of forming limit points of Ti21S ST and Ti21S STA are plotted in Fig. 5. The formability of specimens strained along transverse direction (T) is lower than that along the rolling direction (L) no matter Ti21S ST or Ti21S STA. There is a huge difference between the formability of Ti21S ST and Ti21S STA. The low tensile strength and large elongation lead to high formability. The Ti21S STA has excellent tensile strength while has poor elongation, as well as poor formability.

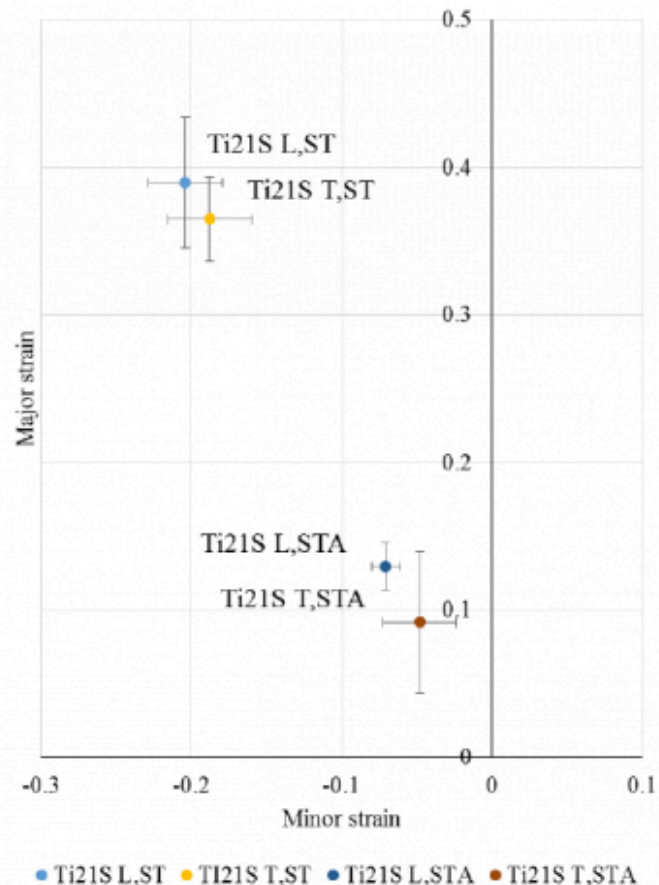

Fig. 5 The forming limit points of Ti21S alloy at various conditions.

\subsection{Simulation}

A finite element (FE) model of the M-K analytical model is built with the ABAQUS software by referring to the model proposed by Zhang [12]. As shown Fig. 6 a, the concept of the M-K model is based on a sheet with an initial thickness $e_{0}{ }^{a}$ (zone a) in which is defined an imperfection materialized by a central groove located symmetrically in the sheet thickness. The thickness of the sheet at the groove (zone b) is noted $e_{0}^{b}$. Here, the direction of the groove is kept perpendicular to the direction of the major strain (direction 1, Fig. 6 a). The initial imperfection factor is defined as $f_{0}$ $=e_{0}{ }^{b} / e_{0}{ }^{a}$. Different displacements $(u, v)$ are applied on the boundaries, respectively along the directions 1 and 2 , for simulating different strain paths. 

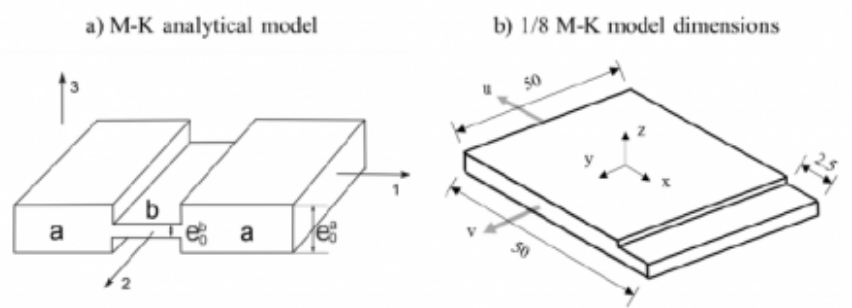

Fig. $6 \mathrm{M}-\mathrm{K}$ model applied in simulation to predict the forming limit curve of Ti21S alloy.

Fig. 6 b shows the dimensions of the FE model used in this study. For reasons of symmetry, only one eighth of the model is defined. The model is meshed by hexahedral elements with an approximate global size of $2 \mathrm{~mm}$. The influence of the groove orientation on the predictive left hand side of the forming limit diagram is not considered in this study. Influence of anisotropy on the results remains very low for this material.

The Ludwick law is chosen to model the hardening behavior of the Ti21S [13]:

$$
\bar{\sigma}=\sigma_{0}+K \bar{\varepsilon}^{n}
$$

Where $\bar{\sigma}$ is the equivalent stress, $\bar{\varepsilon}$ is equivalent plastic strain. $\sigma_{0}, K, n$ are material parameters calibrated from the experimental curve $\bar{\sigma}=f(\vec{\varepsilon})$ obtained from a uniaxial tensile test for the considered conditions (Fig. 4 a). Table 3 presents the values of these material parameters for Ti21S L,ST and Ti21S L,STA. The choice of the hardening law, especially the value of the slope near the necking limit strains, can have an influence on the predictive results. The predictive hardening behavior with the Ludwick's law is very good for moderate and high strains.

Table. 3 Parameters of Ludwick's hardening law for Ti21S L,ST and Ti21S L,STA.

\begin{tabular}{cccc}
\hline Specimen & $\sigma_{0}(\mathrm{MPa})$ & $K(\mathrm{MPa})$ & $n$ \\
\hline Ti21S L,ST & 750 & 481 & 0.4 \\
\hline Ti21S L,STA & 1130 & 628 & 0.46 \\
\hline
\end{tabular}

For Ti21S STA, an isotropic von-Mises yield criterion is assumed, while for Ti21S ST, an anisotropic quadratic yield criterion (Hill48) is considered:

$$
f(\sigma)=\sqrt{F\left(\sigma_{22}-\sigma_{33}\right)^{2}+G\left(\sigma_{33}-\sigma_{11}\right)^{2}+H\left(\sigma_{11}-\sigma_{22}\right)^{2}+2 L{\sigma_{23}}^{2}+2 M \sigma_{31}{ }^{2}+2 N{\sigma_{12}}^{2}}
$$

Where $\sigma_{i j}$ is the stress components, $F, G, H, L, M$, and $N$ are the material parameters defined by anisotropy parameters $R_{11}, R_{12}, R_{22}, R_{13}, R_{23}$ and $R_{33}$. These values are calculated from Lankford coefficients $r_{\theta}$ through the formulations 
below when assuming $R_{11}=R_{13}=R_{23}=1$ :

$$
R_{22}=\sqrt{\frac{r_{90}\left(r_{0}+1\right)}{r_{0}\left(r_{90}+1\right)}}, R_{33}=\sqrt{\frac{r_{90}\left(r_{0}+1\right)}{r_{0}+r_{90}}} \text { and } R_{12}=\sqrt{\frac{3 r_{90}\left(r_{0}+1\right)}{\left(r_{0}+r_{90}\right)\left(2 r_{45}+1\right)}}
$$

Then the anisotropy parameters calculated of Ti21S ST are: $R_{11}=1, R_{12}=1.17, R_{22}=1.03, R_{13}=1, R_{23}=1$ and $R_{33}=1.04$.

The imperfection factor $f_{0}$, is calibrated in order to predict the forming limit point corresponding to the experimental one obtained from the uniaxial tensile test for each material. Then the whole FLC can be predicted with a constant imperfection factor $f_{0}$ for Ti21S ST and for Ti21S STA. The results are shown in Fig. 7 for the different conditions.
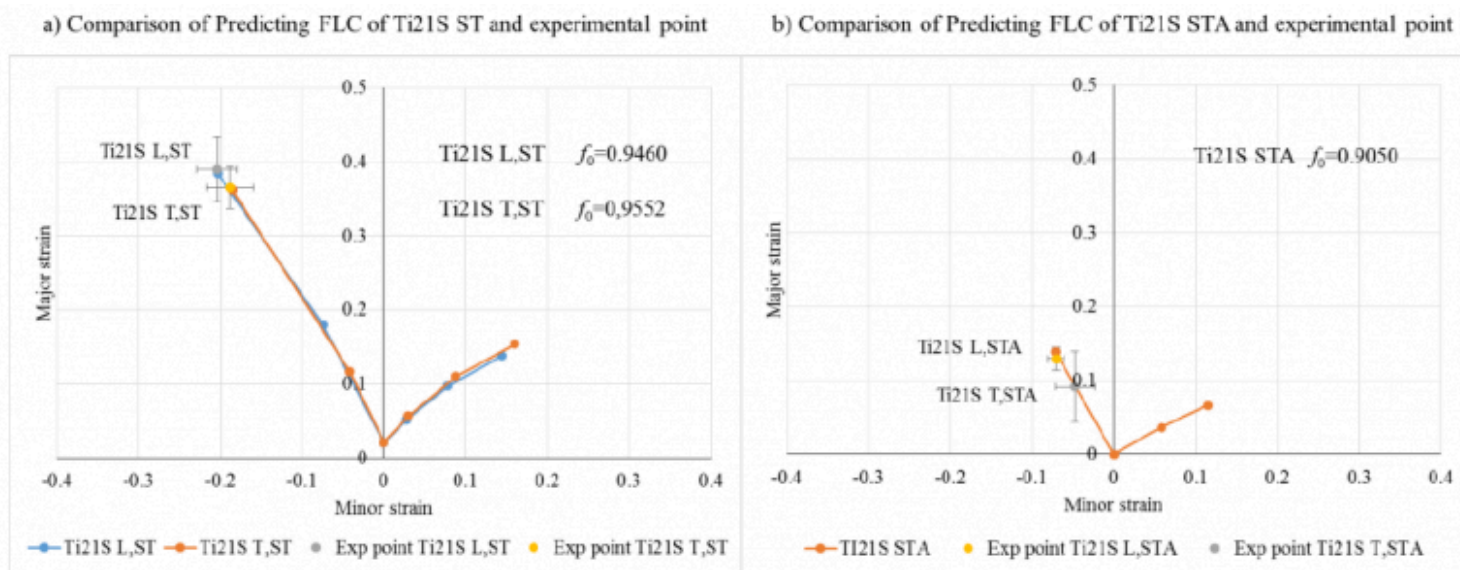

Fig. 7 The predicting FLC and the experimental point of Ti21S ST and Ti21S STA conditions.

With this method, $f_{0}$ values obtained for Ti21S L,ST, Ti21S T,ST and Ti21S STA are respectively 0.9460, 0.9552 and 0.9050. Each value of $f_{0}$ represents the feature of the corresponding microstructure. It can be considered as a parameter of the material. The comparison of $f_{0}$ values of ST and STA conditions (Fig. 7 a and Fig. 7 b) shows that solution treatment state (Ti21S ST) leads to a larger imperfection factor $f_{0}$ (less defects) than aged state (Ti21S STA). The calibration of the imperfection on the longitudinal (Ti21S L,ST) and transverse (Ti21S T,ST) directions leads to fairly close imperfection factors (0.946 and 0.9552). These values are consistent with the experimental values of the limit strains (slightly higher formability for the longitudinal direction compared to the transverse one). On the right part of FLC, a smaller $f_{0}$ reduces the formability. For all the predictions, the formability of the plane strain state is very low.

\subsection{Fracture analysis}

SEM observations are conducted on the fracture surface of tensile specimens for each condition. Fractographies of Ti21S ST (L and T) specimens show a fracture with a flat surface with large size dimples (Fig. 8 a and b), showing a transgranular fracture. Fractographies of Ti21S STA specimens conversely show very small and shallow dimples with some visible grains (Fig. $8 \mathrm{c}$ and d). The fracture is then intergranular in Ti21S STA specimens. That is why STA specimens have a lower ductility. This change in fracture mechanism after ageing treatment is clearly due to the precipitation of $\alpha$ phase. 


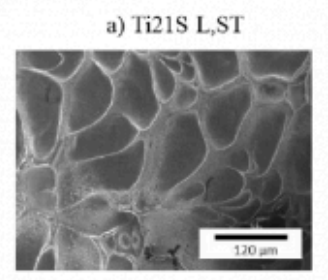

c) Ti21S L,STA

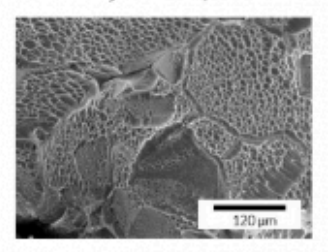

b) Ti21S T,ST

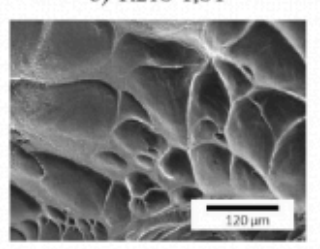

d) Ti21S T,STA

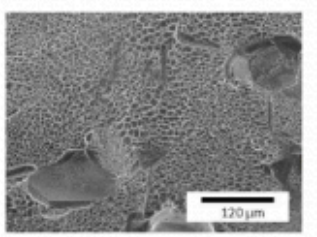

Fig. 8 SEM fractographies of Ti21S ST and Ti21S STA specimens.

\section{Conclusions}

The effect of microstructures on the formability of Ti21S has been investigated. Several conclusions can be drawn:

1. The formability calculated for the tensile specimens along the longitudinal direction is better than for the transverse direction. The aging treatment (STA) drastically lowers the formability compared to the solution treatment state (ST). This phenomenon can be attributed to the precipitation of $\alpha$ phase in the STA condition.

2. The simulation with M-K model can predict FLC with the effect of microstructure. The Ti21S ST has a larger imperfection factor $f_{0}$ than Ti21S STA. We can suppose that the material Ti21S ST in the solution treatment state (ST) has less defect than the aged state (STA) when considering the formability. Meanwhile, the imperfection factor in the longitudinal direction is less than the transverse direction.

3. The Ti21S ST has a transgranular fracture and large size dimples, showing a ductile fracture, which is beneficial to the formability. The Ti21S STA has an intergranular fracture and small shallow dimples which highlight a fragile fracture inducing a poor formability.

\section{Acknowledgements}

C. Hu acknowledges the China Scholarship Council (CSC) for his Ph.D financial support. The authors also acknowledge the SCANMAT platform of the University of Rennes for providing access to SEM facilities.

\section{Bibliography}

[1] Beal JD, Boyer R, Sanders D. Forming of titanium and titanium alloys. Metalworking: Sheet Forming (ASM Handbook). 2006;14:656-69.

[2] Goodwin GM. Application of strain analysis to sheet metal forming problems in the press shop. Sae Transactions. 1968 Jan 1:380-7.

[3] ASTM E2218-15. Standard test method for determining forming limit curves. 2008. 
[4] Nakazima K, Kikuma T, Hasuka K. Study on the formability of steel sheets. YAWATA TECH REP, SEPT. 1968,--264--, 8517-8530. 1968.

[5] Marciniak Z, Kuczyński K, Pokora T. Influence of the plastic properties of a material on the forming limit diagram for sheet metal in tension. International Journal of Mechanical Sciences. 1973 Oct 1;15(10):789-800.

[6] Marciniak Z, Kuczyński K. Limit strains in the processes of stretch-forming sheet metal. International journal of mechanical sciences. 1967 Sep 1;9(9):609-20.

[7] Chamos AN, Labeas GN, Setsika D. Tensile behavior and formability evaluation of titanium-40 material based on the forming limit diagram approach. Journal of materials engineering and performance. 2013 Aug 1;22(8):2253-60.

[8] Liu DK, Huang GS, Gong GL, Wang GG, Pan FS. Influence of different rolling routes on mechanical anisotropy and formability of commercially pure titanium sheet. Transactions of Nonferrous Metals Society of China. 2017 Jun 1;27(6):1306-12.

[9] Froes FH, Bomberger HB. The beta titanium alloys. JOM. 1985 Jul 1;37(7):28-37.

[10] Chamos AN, Labeas GN, Setsika D. Tensile behavior and formability evaluation of titanium-40 material based on the forming limit diagram approach. Journal of materials engineering and performance. 2013 Aug 1;22(8):2253-60.

[11] Sun F, Prima F, Gloriant T. High-strength nanostructured Ti-12Mo alloy from ductile metastable beta state precursor. Materials Science and Engineering: A. 2010 Jun 25;527(16-17):4262-9.

[12] Zhang C, Leotoing L, Zhao G, Guines D, Ragneau E. A methodology for evaluating sheet formability combining the tensile test with the M-K model. Materials Science and Engineering: A. 2010 Nov 25;528(1):480-5.

[13] Ding J, Zhang C, Chu X, Zhao G, Leotoing L, Guines D. Investigation of the influence of the initial groove angle in the M-K model on limit strains and forming limit curves. International Journal of Mechanical Sciences. 2015 Jul 1;98:59-69.

PDF automatically generated on 2021-05-20 08:29:13

Article url: https://popups.uliege.be/esaform21/index.php?id=1630

published by ULiège Library in Open Access under the terms and conditions of the CC-BY License (https://creativecommons.org/licenses/by/4.0) 